\title{
Nutrigenomics and Nutrigenetics: New Insight in Disease Prevention and Cure
}

\author{
Praveen Sharma $^{1} \cdot$ Shailendra Dwivedi $^{1}$
}

Published online: 22 September 2017

(C) Association of Clinical Biochemists of India 2017

The perception that diet influences health is an ancient one and association between nutrient and diseases had been established in past, which is now being explored through modern epidemiological studies. Environmental factors (including food nutrients), and genetic makeup play an important role in phenotypic appearance of a trait via central dogma of biology. Environmental factors include various exposure factors that come in contact by oral intake, air and via skin commonly. Several bioactive food components, including both essential and non-essential nutrients, can regulate gene expression patterns. It is conceivable that with the alteration in the food habits and life styles, people are becoming more disposed to diet related diseases and disorders. Relentless progress in molecular biology and techniques over the past decades now resolve the association of nutrients and diseases at the personalized levels [1-3]. Thus, Nutrigenomics is providing the effects of ingested nutrients and other food components on gene expression and gene regulation, i.e., diet-gene interaction in order to identify the dietetic components having beneficial or detrimental health effects. Like Phenylketonuria (PKU) an inborn error of metabolism which is caused by a change (mutation) in a single gene. Diagnosed individuals have to avoid food containing the amino acid phenylalanine.

Recent advances in the fields of functional foods, nutraceuticals and its impact on human health have been concluded into modern molecular nutrition with a more precise term of "Nutrigenomics or Nutrigenetics".

Praveen Sharma

praveensharma55@gmail.com

1 Department of Biochemistry, All India Institute of Medical Sciences, Jodhpur 342005, India
Nutrigenomics as a new and emerging field of genomics which comprises the analysis of effect of nutrient intake on the whole genome (complete genetic make-up; including epigenetic changes), the proteome (the sum total of all proteins), and the metabolome (the sum of all metabolites). For example, Galactosemia is a disease that results from an inherited genetic deficiency of one of the three enzymes that are implicated in the metabolism of galactose.

In contrary, Nutrigenetics includes the study of individual differences at the genetic level that sways individual responses to diet. These individual differences may be at the level of single nucleotide polymorphisms rather than at the gene level. Thus it emphasizes to clarify the different response of the gene variability (of different individuals) to the same nutrient. Methylene-tetra-hydro folate reductase gene (MTHFR) is a well-known example of nutrigenetics presenting a gene-nutrient interaction. Specifically, this enzyme is required for conversion of 5, 10-methylenetetrahydrofolate to a molecule called 5-methyltetrahydrofolate. This reaction is required for the multistep process that converts the amino acid homocysteine to another amino acid, methionine. MTHFR is involved in the metabolism of folic acid and maintenance of the normal blood level of homocysteine. A particular MTHFR gene SNP (C677T and A1298C) is associated with elevated homocysteine levels of in the blood of carriers, especially if there is a dietary deficiency of folic acid. Individuals with low activity of the MTHFR enzyme may present with elevated homocysteine levels, which have been associated with inflammation and heart disease, birth defects, difficult pregnancies, and potentially an impaired ability to detoxify. Nutrient deficiencies in Folate, B6 and B12 have been associated with elevated homocysteine.

The basic elements of nutritional genomics includes: (1) diet is supposed to be a main predisposing factor for 
several diseases in some individuals under certain conditions. Like saturated fat containing food should be avoided in CVD. (2) Diet entities, nutrients change the gene structure or gene expression, and subsequently, the human genome. Like diet contains several precursors methionine, folate, choline, betaine and vitamins $\mathrm{B}_{2}, \mathrm{~B}_{6}$ and $\mathrm{B}_{12}$, that are important precursors of synthesis of SAM (S-Adenosylmethionine) which is universal methyl donor during methylation, reduced availability of methyl donors should result in low SAM synthesis and global DNA hypomethylation, and vice versa. (3) The genetic variants between individuals can explain the equilibrium between health and disease. Several genotypes/haplotypes have been explored in various diseases that confer more susceptibility of the disease than others. (4) Genes that are reliant on dietary factors in its regulation may have a role in the initiation, advancement, and progression of chronic diseases. This last elements of the nutrigenomics have certain ethical barriers to explore in human subjects but in vivo mice study has proven that miR-483-3p has role in development of type 2 diabetes or regulation of metabolic health and also in obese subject it is responsible to deposit more fats in adipose tissue [4].

Thus, overall objectives of this emerging omics is to explore how genes interact with nutrients. It elucidates that how DNA and genetic code influence our need for specific nutrients and also within specific quantities are required for maintaining good health throughout life. It is helpful to determine the personalized diet that means individual nutritional requirements based on the genetic makeup of the person as well as clarifies some etiological perspectives of chronic diseases like type- 2 diabetes, cancer, obesity and cardiovascular diseases (CVD) because of associations between diet and chronic diseases.

Dietary fat is a significant factor in the development of the Metabolic Syndrome (MetS). High-fat diets, especially rich in saturated fatty acid (SFA) diets, have been confirmed to play a pivotal role in development of adiposity, inflammation and insulin sensitivity, promoting the development of insulin resistance, the MetS and Type 2 Diabetes Mellitus (T2DM). It is reported that diet with high content of monounsaturated fatty acids (MUFA) have been linked with enhancements in insulin sensitivity in healthy subjects. Recent study on large pan-European isocaloric dietary intervention study of MetS subjects (LIPGENE study) reported that replacement of SFA with either MUFA or low-fat, high-complex carbohydrate to enhance insulin sensitivity was only successful in subjects whose daily preintervention dietary fat consumption was below the median $(<36 \%$ energy from fat) [6]. Similarly, the study conducted on MUFA in obesity demonstrated that MUFA rich diets had favorable effects on insulin and glucose concentrations and were linked with reduced body fat regain
[7]. Moreover, animal studies have verified favorable effects of long chain $n-3$ polyunsaturated fatty acids (LC $n-3$ PUFA) on inflammation and insulin sensitivity, translation of these potency of anti-diabetic effects in humans has been found very difficult due to conflicting epidemiological data in relation to their influence on insulin resistance in humans $[5,7]$.

Similarly, it is estimated that diet influences about $30-40 \%$ of all cancer cases, many studies indicate that breast, prostate, liver, colon and lung cancers are linked to the dietary intakes [1]. One interesting example of the complex interactions between genetics, diet and disease is from a study on the occurrence of hepatocellular carcinoma in Sudanese population. It was perceived that a stronger relationship existed between the risk of developing the disease and the consumption of peanut butter contaminated with aflatoxins in Sudanese people with the glutathione $S$-transferase M1 null genotype compared to those lacking this genotype. So far, more than 1000 different phytochemicals have been recognized with cancer preventive activities. Several dietary compounds such as selenium, epigallocatechin-3-gallate, phenylethyl isothiocyanate, retinoic acid, sulforaphane, curcumin, apigenin, quercetin and resveratrol have their cancer preventive effect by apoptosis inhibition. During chronic inflammation, several changes have been observed in cells including increased DNA damage, disruption of DNA repair pathways, cellular proliferation, inhibition of apoptosis, and promotion of angiogenesis and invasion [1, 8]. Cancer metabolism has unique attention due to its involvement in every stage of cancer development. Cancer cells are characterized by an altered metabolism to maintain their rapid enormous growth. Omega- 6 and omega- 3 PUFA have been proven to differentially influence gene expression linked to inflammation (e.g. TNF $\alpha$, IL-1 $\beta$, IL-6,IL-18), angiogenesis (e.g. VEGF, PDGF, IGF-1, MMP-2), and proliferation (e.g. cyclin, p53, Wnt, PTEN) and can thereby control tumorigenesis. The crucial role of nutrigenomics in the field of cancer therapy still needs more research $[1,9]$.

Thus, Nutrigenomics will help in evaluating the individual nutritional needs based on the genetic profile of the individual (personalized diet) as well as help in curing and preventing the chronic diseases. Therefore, there is a serious need to enhance more research in this arena to support people in understanding the association between diet and health, so that everyone should be benefitted from the genomic revolution.

\section{References}

1. Dwivedi S, Shukla S, Goel A, Sharma P, Khattri S, Pant KK. Nutrigenomics in breast cancer. In: Barh $\mathrm{D}$, editor. Omics 
approaches in breast cancer. New Delhi: Springer; 2014. p. 127-51.

2. Sharma P, Dwivedi S. Prospects of molecular biotechnology in diagnostics: step towards precision medicine. Indian J Clin Biochem. 2017;32(2):121-3.

3. Dwivedi S, Purohit P, Misra R, Pareek P, Goel A, Khattri S, et al. Diseases and molecular diagnostics: a step closer to precision medicine. Indian J Clin Biochem. 2017;. doi:10.1007/s12291-0170688-8.

4. Ferland-McCollough D, Fernandez-Twinn DS, Cannell IG, et al. Programming of adipose tissue miR-483-3p and GDF-3 expression by maternal diet in type 2 diabetes. Cell Death Differ. 2012;19(6):1003-12. doi:10.1038/cdd.2011.183.

5. Tierney AC, McMonagle J, Shaw DI, Gulseth HL, Helal O, Saris $\mathrm{WH}$, et al. Effects of dietary fat modification on insulin sensitivity and on other risk factors of the metabolic syndrome-LIPGENE: a European randomized dietary intervention study. Int $\mathrm{J}$ Obes (Lond). 2011;35:800-9.

6. Due A, Larsen TM, Hermansen K, Stender S, Holst JJ, Toubro S, et al. Comparison of the effects on insulin resistance and glucose tolerance of 6-mo high-monounsaturated-fat, low-fat, and control diets. Am J Clin Nutr. 2008;87:855-62.

7. Phillips CM. Nutrigenetics and metabolic disease: current status and implications for personalised nutrition. Nutrients. 2013;5(1):32-57. doi:10.3390/nu5010032.

8. Ardekani AM, Jabbari S. Nutrigenomics and cancer. Avicenna J Med Biotechnol. 2009;1(1):9-17.

9. Dwivedi S, Shukla KK, Gupta G, Sharma P. Non-invasive biomarker in prostate carcinoma: a novel approach. Indian J Clin Biochem IJCB. 2013;28(2):107-9. 